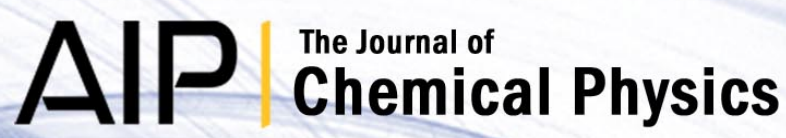

\section{Coarse-grained modeling of interactions of lipid bilayers with supports}

Matthew I. Hoopes, Markus Deserno, Margie L. Longo, and Roland Faller

Citation: J. Chem. Phys. 129, 175102 (2008); doi: 10.1063/1.3008060

View online: http://dx.doi.org/10.1063/1.3008060

View Table of Contents: http://jcp.aip.org/resource/1/JCPSA6/v129/i17

Published by the American Institute of Physics.

\section{Additional information on J. Chem. Phys.}

Journal Homepage: http://jcp.aip.org/

Journal Information: http://jcp.aip.org/about/about_the_journal

Top downloads: http://jcp.aip.org/features/most_downloaded

Information for Authors: http://jcp.aip.org/authors

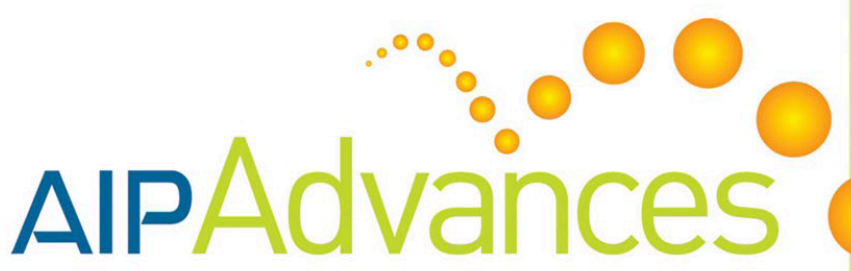

Explore AIP's new open-access journal

Article-level metrics now available

Join the conversation!

Submit Now 


\title{
Coarse-grained modeling of interactions of lipid bilayers with supports
}

\author{
Matthew I. Hoopes, ${ }^{1}$ Markus Deserno, ${ }^{2}$ Margie L. Longo, ${ }^{3,1}$ and Roland Faller ${ }^{1,3, a)}$ \\ ${ }^{1}$ Graduate Group in Biophysics, University of California, Davis, California 95616, USA \\ ${ }^{2}$ Department of Physics, Carnegie-Mellon University, Pittsburgh, Pennsylvania 15213, USA \\ ${ }^{3}$ Department of Chemical Engineering and Materials Science, University of California, \\ Davis, California 95616, USA
}

(Received 15 July 2008; accepted 7 October 2008; published online 5 November 2008)

\begin{abstract}
We characterize the differences between supported and unsupported lipid bilayer membranes using a mesoscopic simulation model and a simple particle-based realization for a flat support on to which the lipids are adsorbed. We show that the nanometer roughness of the support affects membrane binding strength very little. We then compare the lipid distributions and pressure profiles of free and supported membranes. The surface localization of the proximal leaflet breaks the symmetry seen in a free bilayer, and we quantify the entropic penalty for binding and the increased lateral compression modulus. (C) 2008 American Institute of Physics. [DOI: 10.1063/1.3008060]
\end{abstract}

\section{INTRODUCTION}

Supported lipid membranes are an important research platform for static and dynamic physical measurements of a fluid bilayer and its constituents. ${ }^{1,2}$ Their extensive use as a model system has provided data such as lipid diffusion constant ${ }^{3,4}$ phase behavior, ${ }^{5}$ and the association of other biological molecules such as proteins ${ }^{6}$ and sugars. ${ }^{7,8}$ Even interactions with whole cells have been studied. ${ }^{9,10}$ Characterization of supported lipid bilayers has been accomplished with numerous methods including atomic force microscopy, ${ }^{11}$ $\mathrm{x}$-ray and neutron scattering and reflectometry, ${ }^{12-15}$ nuclear magnetic resonance, ${ }^{16}$ quartz crystal microbalance, ${ }^{17}$ surface plasmon resonance, ${ }^{18-21}$ ellipsometry, ${ }^{6}$ electrical impedance spectroscopy, ${ }^{15}$ and many versions of fluorescence microscopy such as fluorescence resonance energy transfer, ${ }^{22}$ fluorescence recovery after photobleaching, ${ }^{23}$ fluorescence interference contrast, ${ }^{24,25}$ fluorescence correlation spectroscopy, ${ }^{26}$ and total internal reflection fluorescence. ${ }^{27}$

Phospholipid bilayers adsorb to hydrophilic surfaces to create a supported bilayer leaving a layer of water up to $5 \AA$ thick - in addition to the hydration shell of the head groups-between the substrate and the head groups of the lipids in the proximal (closest to the solid) leaflet. ${ }^{12,13}$ Its thickness and structure depend on surface preparations and lipid type and it has been shown to be vanishingly small or even undetected in some cases. ${ }^{12}$ This thin layer of water does not provide enough room for the incorporation of transmembrane proteins that extend beyond the bilayer on the proximal side and reduces the mobility of molecules in the membrane. To address this problem several methods have been developed to increase the spacing between the bilayer and substrate. ${ }^{15,18,28-30}$ Nonetheless, measurements of pure and mixed lipid membranes performed directly on supports remain a valuable source of information on the nature of lipid-lipid and lipid-solvent interactions.

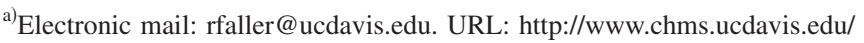
faculty/faller.php.
}

The effects induced on adsorbed lipid bilayers by a surface can be systematically studied in molecular simulations since this method provides access to many local details that are very hard to measure in experiments. Surprisingly, although computational studies of phospholipid bilayers are abundant, supported membranes have received little attention until very recently. ${ }^{31-34}$ Including a solid surface in simulations provides several exciting opportunities for understanding the specific and generic aspects of the interaction between bilayer and surface. Obvious questions deal with the balance between adsorption and deformation energies or structural changes to the membrane as a result of the reorganization of lipids imposed by the proximity of a solid.

In order to address this large spectrum of physical phenomena in simulations, it is necessary to adopt multiscale modeling techniques and choose a level of resolution suitable for the question being asked. In this work we use a coarse-grained (CG) model that employs a three particle representation of the lipid and no explicit solvent; the generic interaction forces on this level of granularity are applicable to a wide range of different types of lipid systems. The intrinsic speed-up of coarse-graining as well as the reduced number of particles is ideally suited to focus on collective phenomena of a large number of molecules with high statistical significance. For instance, our model cannot look into subtle aspects of the poorly understood confined water layer between bilayer and substrate simply because it does not even represent water. However, one can plausibly argue that generic questions on a larger scale-such as symmetry breaking, confinement induced lipid order, or changes in elastic moduli-are largely independent of details of such a layer. It is this latter type of questions that the present article is dedicated to.

\section{COMPUTATIONAL DETAILS}

\section{A. Coarse-grained modeling of biomembranes}

Several highly coarse-grained solvent-free bilayer models have recently been developed ${ }^{35-39}$ and any one of them 
might be used in studies of generic aspects of supported membranes. Choices of which model to use may depend on the resolution needed to analyze the properties of interest. Here we use the model developed by Cooke and Deserno, which we implement using the ESPRESSO Molecular Dynamics package. ${ }^{40}$ This model has been shown to lead to a selfassembling fluid bilayer that reproduces important mechanical properties such as compressibility and bending modulus ${ }^{41}$ with values in the experimentally relevant ranges. We measure length, energy, and time in units of $\sigma$ (diameter of lipid tail beads and surface beads), $\varepsilon$ (depth of cohesion potential between lipid tail beads), and $\tau=\sigma \sqrt{m / \varepsilon}$ ( $m$ is the bead mass which is the same for all beads), respectively. Their relation to real units can be established as follows: Since for a free membrane we find a thickness of about $5 \sigma$, comparison with a typical experimental value of $5 \mathrm{~nm}$ shows that $\sigma \approx 1 \mathrm{~nm}$. Our temperature is chosen as $k_{B} T=1.1 \varepsilon$, and since at room temperature $k_{B} T \approx 4.1 \times 10^{-21}, J \approx 0.6 \mathrm{kcal} / \mathrm{mol}$, this fixes $\varepsilon \approx 0.54 \mathrm{kcal} / \mathrm{mol}$. Together with an interaction range of $w_{c}=1.6 \sigma$ for the $\cos ^{2}$ tail attraction length this sets the resulting membranes into the fluid $L_{\alpha}$ phase. Finally, by mapping the diffusion constant of lipids in a free membrane to an experimental value of about $1 \mu \mathrm{m}^{2} / \mathrm{s}$, we find $\tau \approx 10 \mathrm{~ns}$. The latter mapping is admittedly very rough but since in the present study we only look at static properties, the precise value of $\tau$ is immaterial. For details of the interactions the reader is referred to the original definition of the model. ${ }^{35}$

Since the substrate onto which we will adsorb membranes consists of a hexagonal lattice of beads, it proves convenient to adopt the following convention for specifying the lateral dimension of our simulation box: Assume that we place $A$ surface beads in a row at mutual distance $d$. The next row stacked onto this has a perpendicular distance of $\frac{1}{2} \sqrt{3} d$. To enable periodic boundary conditions, we need to have an even number of rows, say $C$. Defining $B=$ floor $\left(\frac{1}{2} \sqrt{3} C\right)$, we call this an $A \times B$ system. Unless otherwise stated, we choose the bead distance $d=\sigma$.

The density profile calculations used $50 \times 50$ bilayers averaged over $30000 \tau$ with 4172 lipids. Broadening of density profiles due to bilayer undulations was reduced by defining its midplane individually within the squares of a $16 \times 16$ grid. Fluctuations were reduced in the stress profile calculations by using a smaller $20 \times 20$ bilayer and 690 lipids, with pressure averaged over $30000 \tau$. For the tension and area per molecule calculations below, every simulation spanned $10000 \tau$ in a $20 \times 20$ bilayer without the fluctuation compensation mentioned above.

Comparisons between free and supported bilayers were made by first simulating a free system in an $\mathrm{N} \Sigma \mathrm{T}$ ensemble (constant number of lipids, lateral tension, and temperature; the simulation box height normal to the bilayer is fixed). From the lateral box size at equilibrium, an area per lipid was calculated. For the spacing of the periodic solid support to extend properly over periodic boundary conditions and its strength not to depend on simulation time, the simulation box size must remain fixed. Subsequent simulations therefore used an NVT ensemble using the surface construction described above. The number of lipids was chosen to repro-

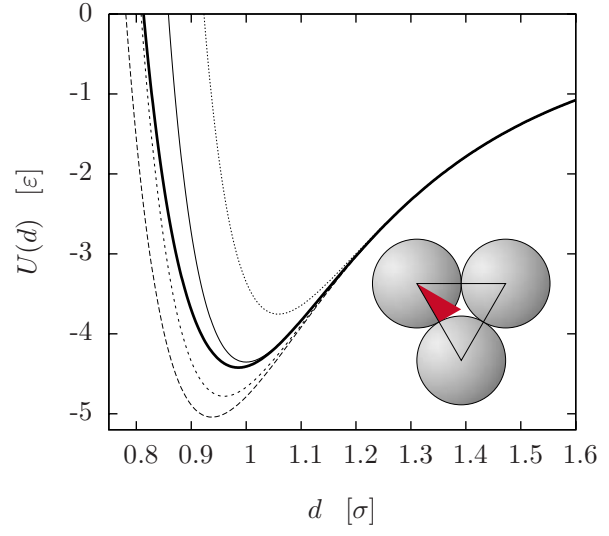

FIG. 1. (Color online) Interaction energy as a function of vertical position for a lipid head group placed over the surface of a hexagonally corrugated substrate at the three vertices of twofold (short dashed), threefold (long dashed), and sixfold (dotted) symmetries. The asymptotic large-distance potential (solid) from Eq. (1) and the numerically integrated effective potential (bold solid) are shown as well. The inset illustrates three surface particles with the irreducible area shaded.

duce the desired area per lipid. In all calculations, periodic boundaries in all three dimensions were used.

\section{B. Details of the implementation of the surface interaction}

All surface particles interact with lipid head beads by a standard Lennard-Jones 12-6 potential (length and energy parameter are $\sigma$ and $\varepsilon$, respectively; cutoff at $r_{c}=2.5 \sigma$ ), while the two tail particles of the lipid and the surface particles interact via a purely repulsive Weeks-Chandler-Andersen ${ }^{42}$ potential. Supported lipid bilayers are formed on hydrophilic surfaces such as glass, quartz, or mica. Common to these surfaces is the high wettability that yields very low contact angles at the air-watersolid triple line. (If surfaces are hydrophobic, lipids tend to form monolayers ${ }^{43}$ with the tails toward the solid surface.) In our studies surface particles are hexagonally packed, their positions are completely fixed, and they interact only with the moving particles. In order to characterize the effect of this discretization on membrane adsorption, we first determined the position-dependent binding potential for a single lipid on this corrugated substrate.

Surfaces made of particles can be viewed as a special case of analytically continuous surfaces with varying degrees of roughness. On a surface of hexagonally packed spheres many points are equivalent as a result of the lattice symmetry of the surface $(p 6 m)$. The shaded triangle in the inset of Fig. 1 represents an irreducible collection of points on the surface which - by translations, rotations and mirroring operationscan create the entire lattice.

To fully sample the adsorption characteristics one may therefore restrict oneself to the prismlike volume extending up from the surface with this triangular area as its base. In the following we will focus on its vertices, which are (stationary) points of twofold, threefold, and sixfold symmetries. To determine the interaction potential at these lateral positions, a lipid head bead was moved normally to the surface. The resulting potentials are shown in Fig. 1. At the point of 

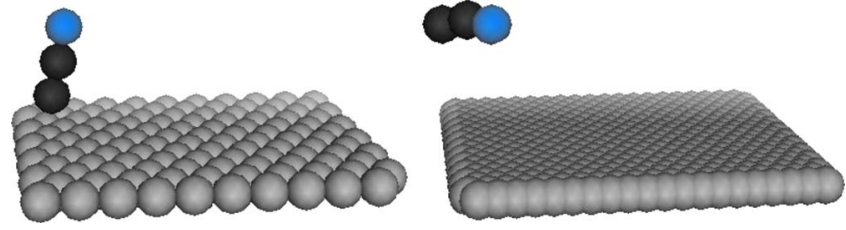

FIG. 2. (Color online) Variable corrugation levels with particle spacings of $\sigma$ (left) and $0.5 \sigma$ (right).

minimum energy for each position, the difference between the two extremes-the points of threefold and sixfold symmetries-was about $1.2 \varepsilon$. Sufficiently far away from the surface one can employ a continuum approximation and easily determine the following analytic expression for the asymptotic interaction potential:

$$
\frac{U_{\text {asymp }}(d)}{\varepsilon}=\frac{16 \pi}{\sqrt{3}}\left\{\frac{1}{10}\left(\frac{\sigma}{d}\right)^{10}-\frac{1}{4}\left(\frac{\sigma}{d}\right)^{4}\right\},
$$

which is also included in Fig. 1. Notice that already for distances beyond $1.2 \sigma$ all four functions-the continuum limit and the potentials at the symmetry vertices-are essentially indistinguishable.

The partition function of an adsorbed lipid on this surface includes the energies on the three curves in Fig. 1, as well as all other intermediate values in the prism volume chosen. The fifth curve in Fig. 1 is the effective potential which was obtained by numerically integrating over the energies a particle experiences in the $x-y$ plane at fixed $z$. Since there is a level of corrugation on our surface as a result of the discrete particles used, we determined how the adsorption energy changes with respect to changes in corrugation. Particles may be laid out on the surface with spacings less than $\sigma$ (recall that these particles do not interact), and in such a case the level of corrugation is reduced due to overlapping spheres, as illustrated in Fig. 2.

In the limit of an infinite number of spheres this leads to a smooth surface. Clearly, reducing the spacing and increasing the number of particles per unit area will increase the energy density of the surface. To first order this can be compensated by rescaling the energy of the individual surface particle. Decreasing the distance between two particles by a factor of $\alpha$ increases the number of particles in a given area by a factor of $\alpha^{2}$. To compensate for this we reduce the interaction energy by a factor $\alpha^{-2}$. This is not exact for finite distances, as the distribution of interaction distances changes and cutoff effects play a role.

Figure 3 shows histograms of interaction energies of a single lipid with surfaces using different surface particle spacing values. We show both results from a Monte Carlo counting procedure, which samples the density of states inside the irreducible prism and multiplies this by the Boltzmann factor, as well as energy histograms obtained by direct molecular dynamics simulations of a single lipid above the corrugated substrate. All histograms diverge at $E$ $=0$ in a nonintegrable way since an infinite phase space above the substrate opens up in the limit $E \rightarrow 0$. While this prevents the normalization of the energy distribution (the partition function does not exist in a strictly infinite half
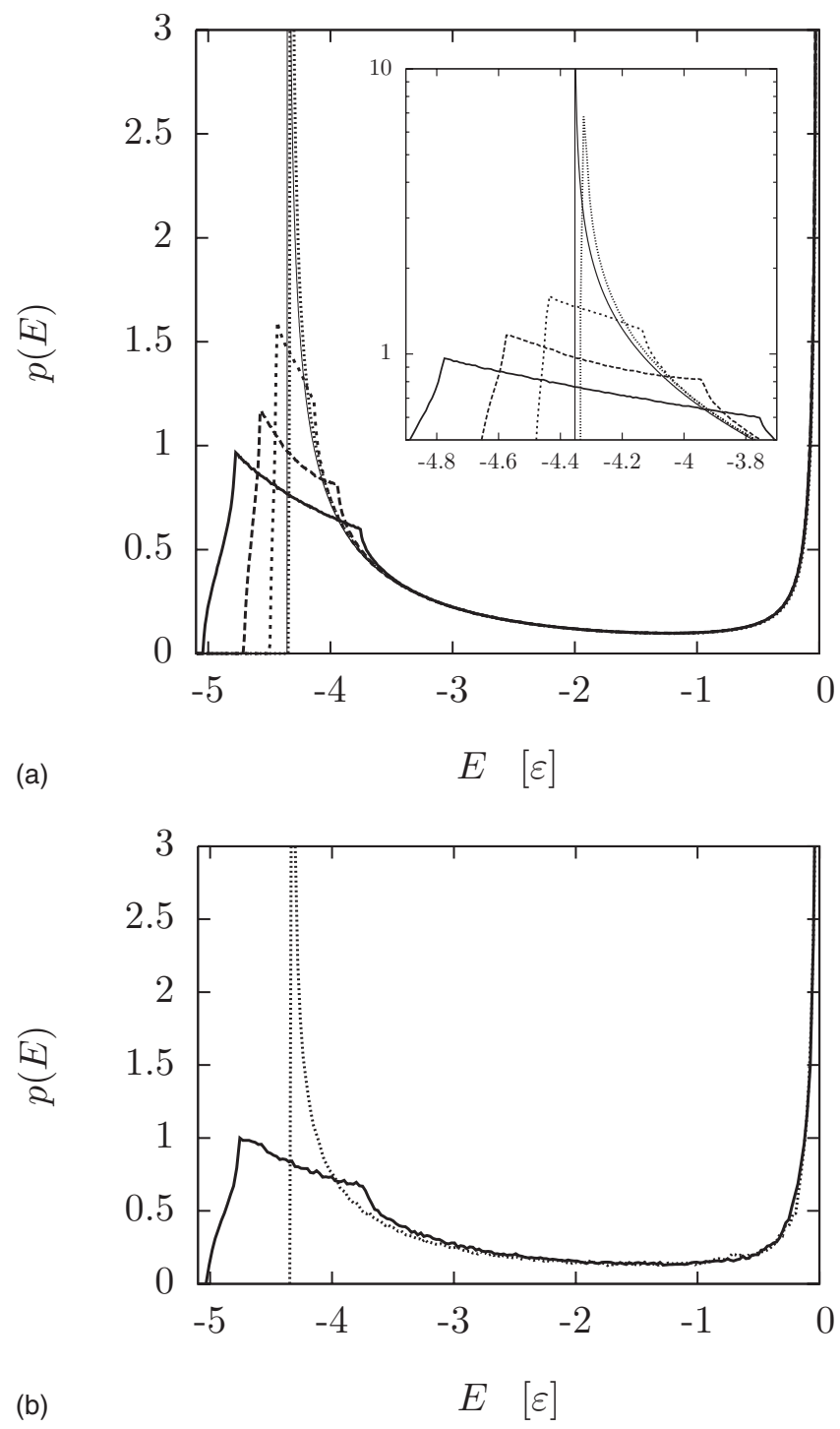

FIG. 3. Energy histograms for single lipid interactions with the surface are shown for Monte Carlo (MC) (top) calculations where particle spacings are $1 \sigma$ (bold solid), $0.875 \sigma$ (long dash), $0.75 \sigma$ (short dash), $0.5 \sigma$ (dotted), and for a continuous surface (solid). For molecular dynamics (bottom) simulations, similar histograms were developed from energy calculations over $7500 \tau$ divided into 200 bins on surfaces with $1 \sigma$ (solid) and $0.5 \sigma$ (dotted) spacing. For this calculation the Lennard-Jones-cutoff has been extended to $5.0 \sigma$ to be more comparable to the MC calculations with no cutoff.

space), relative values remain meaningful. All histograms increase markedly around the binding energy of the potential. The kinks are van Hove singularities in the density of states, corresponding to the flat points of the surface potential. In the limit of a smooth surface they rapidly merge into a single integrable divergence at the binding energy of the flat substrate, $E=(12 \pi / 5 \sqrt{3}) \varepsilon$. What is remarkable, in the present context, is that already at a spacing of $0.5 \sigma$ the histograms are hardly distinguishable, showing that the $0.5 \sigma$-corrugated surface and its asymptotic flat equivalent display essentially the same binding characteristics. This is compatible with our previous finding (see Fig. 1) that even for a spacing of $1 \sigma$ the potential of mean force (the free energy of interaction) is very close to the asymptotic binding potential from Eq. (1), which-since it is strictly one-dimensional-contains no more lateral entropic term. All our studies below will be 
conducted using a spacing for the surface particles of $1 \sigma$.

Taken together, these observations clarify how the surface corrugation affects the binding characteristics, how the dominant effect of area density change can be scaled out, and that higher order effects vanish very rapidly upon reduction in the surface particle spacing. It is therefore not difficult to reach the asymptotic limit. This is relevant-both conceptually and technically-since real surfaces are never perfectly smooth. It should also be pointed out that the sharp features in the histograms will get smoothed out once an entire membrane and not just a single lipid binds to the substrate due to the fluctuations of lipid positions and the impact this has on lipid-surface and lipid-lipid binding energies.

For reasons of numerical efficiency we have applied the usual cutoff of $2.5 \sigma$ to all Lennard-Jones interactions. We have checked that this does not qualitatively change the energy histograms, as displayed in Fig. 3. However, the absence of interactions with laterally distant surface beads reduces the total binding strength-and thus shifts the left end of the histogram-by approximately $0.5 \varepsilon$. Since the precise shape of the binding potential is of secondary importance within our highly coarse-grained simulations, we do not believe this difference to qualitatively affect any of our subsequent results. However, the resulting cutoff-dependent binding energy will matter for our purposes, and it should then be kept in mind that at a cutoff of $2.5 \sigma$ the minimum in the potential of mean force occurs at roughly $U_{\min } \approx-3.8 \varepsilon$, implying that the binding energy is $0.5 k_{B} T$ less than for the nontruncated potential.

Finally, let us explain how we choose the surface-lipid interaction strength. The goal is to model a fluid supported bilayer. In order to do so we have to avoid the two limits of (i) unbinding from the surface and (ii) the liquid-to-gel phase transition. For the parameters used here-including the cutoffs-we find that this goal is achieved: membranes are reliably bound but remain fluid (as determined by the fact that lipids still diffuse laterally). We did not perform a systematic study to map out the corresponding boundaries.

\section{RESULTS}

\section{A. Density profiles}

Most lipid bilayer simulations are performed with water on both sides and in the absence of other molecules so that the environment surrounding the membrane is symmetrical. This entails a concomitant symmetry inside the bilayer. A comparison of the density profile across the bilayer of a free and supported membrane clearly shows that this up-down symmetry is broken by the presence of a solid surface (Fig. 4).

The leaflet proximal to the support shows a significant increase in (local) density and order. The thickness of the bilayer does not change substantially when defined as the peak-to-peak distance between the head groups of the proximal and distal leaflet of the free and supported bilayer, which are $4.44 \pm 0.06 \sigma$ and $4.56 \pm 0.06 \sigma$, respectively. However, the density distributions change, and if we define the thickness as the distance between the outer values at half maxi-
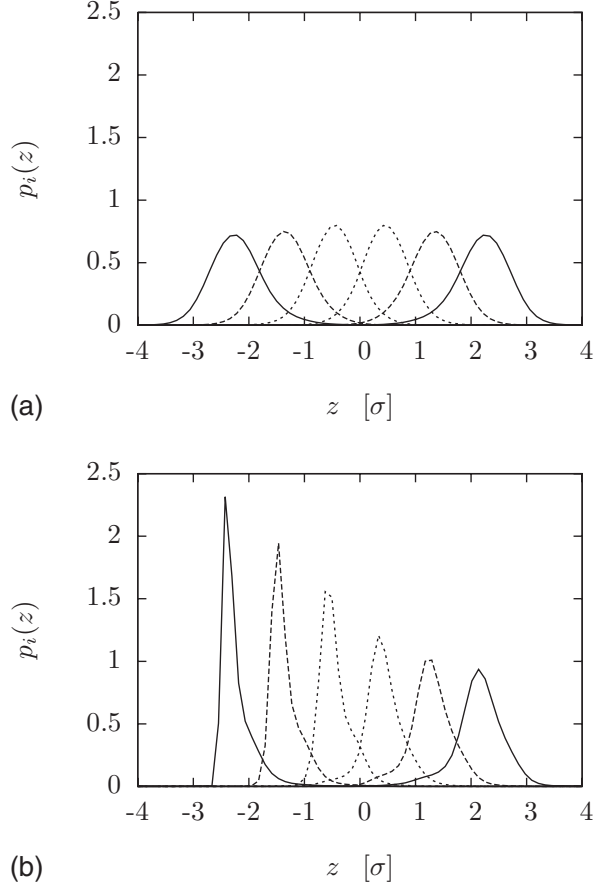

FIG. 4. Density profile for free (top) and supported (bottom) bilayers (the surface is on the left side). Plots show the number density distributions for head groups (solid), middle groups (dashed), and tail groups (dotted) with distances relative to the bilayer center of mass.

mum of the two head group distributions we obtain $5.64 \pm 0.06 \sigma$ and $5.04 \pm 0.06 \sigma$ for the free and supported bilayers. That is, the peaks of density distributions of each superatom for the free and supported bilayer align well, but the shape of the distributions changes. If the external tails of the density distributions are considered, the tail of the proximal leaflet head group distribution is truncated with respect to the free bilayer, implying a $10.6 \%$ reduction in the thus defined bilayer thickness.

Evidence for surface induced flip flop of lipids can also be seen. For the free bilayer, the difference in the total number of lipids per leaflet can be estimated by comparing the integration of the head group density distribution curves for the two leaflets. Using the data in Fig. 4, there is a $0.1 \%$ difference in lipid number between the leaflets in the free bilayer. This is in contrast to the $3.2 \%$ difference in the supported bilayer (this corresponds to four lipids for the free bilayer versus 134 lipids in the supported bilayer). This imbalance is created during a period of enhanced flip flop in the first hundred $\tau$ of the simulation. After this, the rate of flip flop actually decreases below the value of the free bilayer due to the inability for the remaining distal leaflet lipids to enter the higher density proximal leaflet and, conversely, the proximal leaflet lipids to overcome the additional barrier to flip flop created by the surface attraction.

Leaflet interdigitation occurs when the tails of the lipids from one leaflet protrude into the opposing leaflet. This interdigitation is reduced in the supported bilayer, as can be quantified by the $38 \%$ decrease in the overlap integral between the two central density profile peaks in Fig. 4(b) versus Fig. 4(a). Consequently, the manner in which the two leaflets interact is changed by the ordering due to the sup- 
port. Since interdigitation contributes strongly to interbilayer friction, we can expect the viscous coupling between the leaflets of a supported bilayer to be substantially diminished. An associated effect of the ordering of the lipids at the contacting surfaces is the reduction in protrusion modes of the proximal head groups as shown by the sharply truncated tail of their density distribution. This finding agrees with recent simulations as well as experimental data on alcohols and biomembranes, where the disordering induced by alcohols leads to a weak increase in interdigitation. ${ }^{44-46}$

In statistical mechanics every distribution function is associated with a corresponding (von Neumann) entropy. The one-dimensional lipid height distributions $p_{i}(z)$ in Fig. 4 are therefore associated with the entropy

$$
S_{i}=S\left[p_{i}(z)\right]=-k_{B} \int d z p_{i}(z) \ln \left[p_{i}(z) \ell\right],
$$

where $\ell$ is a length that is necessary for dimensional reasons; it affects the value of $S_{i}$ by a constant, but is otherwise arbitrary. For instance, a Gaussian probability density of width $w$ has an entropy $S_{\text {Gauss }}(w) / k_{B}=$ const $+\ln (w)$, showing that doubling the width reduces the free energy by $\Delta F=-T \Delta S$ $=-\ln (2) k_{B} T$. Consequently, we can see that narrowing the bead distributions upon adsorption to a support increases the bilayer's free energy. Since the bead distributions are not independent, their contributions cannot simply be separated and added up. As a (rather conservative) lower bound we will therefore only look at the change in the distribution of the proximal head bead. Using Eq. (2), we find that the total bilayer free energy per proximal lipid is increased by (at least) $\Delta F=0.84 k_{B} T$. Recall that this is exclusively a consequence of normal confinement. There are at least three further effects which lower the entropy of distribution functions and increase the free energy, namely: (i) increase in proximal area density, (ii) increase in (mainly orientational) order, and (iii) the asymmetry in the lipid density across monolayers. Taken together, we see that the order imposed by adsorption entails a significant entropic penalty, showing that the free energy of adsorption is substantially smaller than the energy of adsorption as determined from Eq. (1) or the potentials displayed in Fig. 1. In our case the normal confinement alone diminishes the binding strength by $24 \%$.

\section{B. Pressure profiles}

Following adsorption, changes in the local pressure tensor, specifically the lateral pressure, are seen along the normal of the membrane. This is expected given the modification of the bilayer density discussed above. Since ordering by the substrate reduces fluctuations and protrusions with the greatest effect on the proximal leaflet, one can expect the greatest increase in lateral pressure in the proximal head group region of a supported bilayer for a fixed area compared to the same area for a free bilayer, as can be seen in Fig. 5.

We use the Irving-Kirkwood formulation of the local pressure tensor here. ${ }^{47}$ Note that in our solvent-free coarsegrained model the pressure profiles differ qualitatively from higher resolution models, ${ }^{48,49}$ as the cohesion between leaflets is produced by an attraction between tails and not a

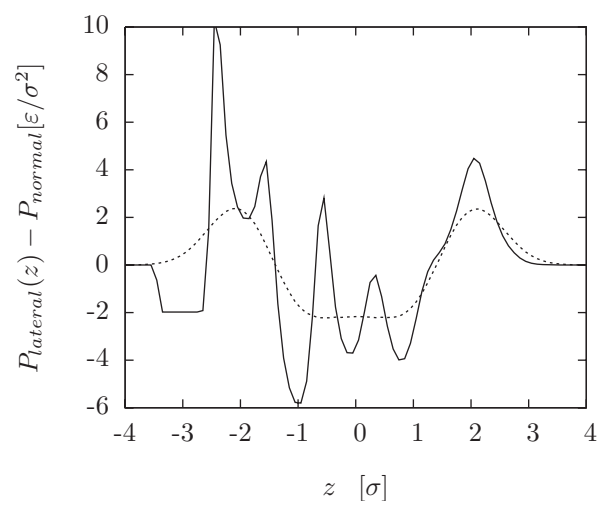

FIG. 5. Pressure profile for $20 \times 20$ free (dotted) and supported (solid) bilayer at constant (zero) tension; the surface is on the left. Distances are relative to the center of mass of the bilayer.

hydrophobic self-assembly. Therefore the bilayer center shows a region of negative tension, induced by the tail attractions, which in atomistic simulations is always positive due to the crowding of tails. However, the overall effects of the support are similar to a recent study of a supported lipid bilayer using a higher resolution model. ${ }^{50}$ We find a pronounced loss of symmetry and can clearly identify the strongest peak directly at the surface. As the density profiles have already indicated, there is significantly higher positional order in the proximal leaflet, leading to effective "interfaces" between "sheets of particles." These effective interfaces also appear in the pressure profile. For obvious reasons the reduced tail interdigitation and especially the low granularity of our CG model overemphasizes the sharpness of pressure and density peaks in the respective profiles, even if individual lipids show realistic fluctuation amplitudes normal to the average bilayer plane. Hence, the negative pressure typically seen in the center of the free membrane is interrupted by peaks of higher pressure seen approximately $0.5 \sigma$ on either side of the bilayer midplane. Note that while the distal leaflet does not attain a complete relaxation from the surface induced ordering transmitted through the bilayer, one can see that the pressure peaks for the head group and midgroup are averaged together enough to prevent a local pressure maximum at the location of the distal midgroup. In a higher resolution model it was found recently that the static structure of the distal leaflet is only very weakly affected by the support. $^{33}$

\section{Area per lipid, tension, and area compressibility}

Values for the area per lipid are usually reported at zero tension. The tension $\gamma$ itself depends on the local pressure distribution and is calculated from the transbilayer integral $^{51,52}$

$$
\gamma=\int d z\left[P_{\text {normal }}-P_{\text {lateral }}(z)\right] .
$$

We emphasize that for fluctuating bilayers one has to be careful precisely what one means by "tension" and "area per lipid." One might either refer to the actual (fluctuating) bilayer area, or to a fictitious projected area, which belongs to the average bilayer surface after fluctuations (up to some 


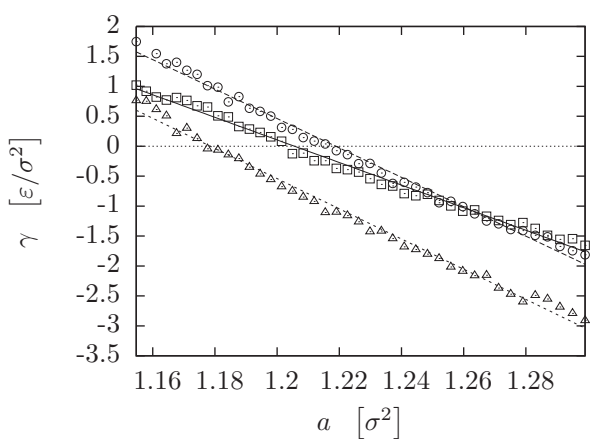

FIG. 6. Tension as a function of area per lipid with trend lines for a free bilayer (squares, solid), a supported bilayer with substrate interactions excluded (circles, dashed), and a supported bilayer with substrate interactions included (triangles, dotted).

upper wavelength cutoff) have been averaged out. As David and Leibler pointed out ${ }^{53}$ the thermodynamic variable canonically conjugate to the mechanical tension is the projected area. In our simulation this is easily identified with the lateral area of the simulation box. Indeed, our barostat (in the free bilayer) couples tension to the lateral box size.

When calculating the local stress tensor in the presence of a substrate, one needs to decide which forces involving the substrate will enter the local stress tensor $\mathbf{P}=-\frac{1}{V} \sum_{i<j}\left\langle\mathbf{r}_{i j} \otimes \mathbf{F}_{i j}\right\rangle$. Since the substrate is considered as infinitely stiff, terms where both $i$ and $j$ correspond to substrate beads will never be included. However, one may choose whether or not to include interaction terms where one bead belongs to the substrate and another to the bilayer. If we do not include such terms, we effectively count exactly the same interactions as in a free bilayer, but its phase space is constraint by the presence of a nearby surface, i.e., we just analyze the effect of flattening out the bilayer. If on the other hand we include these terms, we monitor additionally how the substrate affects the stresses in the bilayer. Below we will refer to this choice as "substrate interactions included or excluded" and show the tension area isotherms in Fig. 6 as triangles and circles, respectively.

When the tension, as determined from Eq. (3), is zero, the area per lipid in a free bilayer is $1.206 \pm 0.002 \sigma^{2}$. Error bars (which are no larger than the markers in Fig. 6) were calculated using box averaging over the last 2500 data points $(\tau)$ of the $10000 \tau$ simulations from which each tension data point was calculated. Using a linear fit to interpolate zero tension area, we fit to the upper and lower limits of the error bars to calculate horizontal error on zero tension area values. Notice that the choice of including or excluding interactions of moving and surface particles gives rise to different values of the tension and thus a different notion of what "zero tension" means for the bilayer state. The zero tension condition determined while excluding the substrate results in an area per lipid of $1.219 \pm 0.002 \sigma^{2}$, while including the substrate interactions gives the smaller value $1.179 \pm 0.002 \sigma^{2}$. In the case of excluded interactions the surface essentially flattens out fluctuations, the bilayer at the thus defined zero tension spreads out and the projected area per lipid increases compared to the free bilayer. However, if we include the surface term, the additional cohesive interactions shift the zero ten- sion point to higher densities, in fact to an area per lipid smaller than in the free bilayer case. The two curves are essentially parallel to each other due to the offset of the surface interaction.

Our two principal results are the following: First, for a real supported membrane under zero tension the area per lipid decreases since, of course, the interactions with the substrate must be included in the calculation of the tension. Second, the slopes of the supported and unsupported tension area isotherms differ significantly. Generally, the (isothermal) lateral expansion modulus $M_{T}$ of a surface is defined as $M_{T}=A(\partial \Sigma / \partial A)_{T}$. For the free bilayer at zero tension it has the value $M_{T \text {,free }}(\sigma=0) \approx 22.7 \varepsilon / \sigma^{2}$. Converting this into physical units we find approximately $85 \mathrm{mN} / \mathrm{m}$, which is slightly lower than typical phospholipid membranes. ${ }^{52}$ However, for supported bilayers this modulus is significantly larger and should not depend on whether the substrate interactions are included or not. If they are excluded, we find $M_{T \text {, bound- }}(\sigma=0) \approx 30.0 \varepsilon / \sigma^{2}$, if they are included we find $M_{T \text {, bound }+}(\sigma=0) \approx 29.7 \varepsilon / \sigma^{2}$; this is on average $30 \%$ stiffer than the free bilayer. The difference between the two can be used as an error estimate. The main reason for the difference to a free membrane is that supported bilayers have fewer undulations, which constitute a softer mode under area change than changing lipid distance itself. Indeed, if the difference in area per lipid was the decisive factor, the supported bilayer system with substrate interactions excluded should be softer than the free bilayer, which it clearly is not.

\section{CONCLUSIONS}

This work provides an analysis of supported lipid bilayers at a highly coarse-grained level. By studying single lipid adsorption we showed how the energy density of the surface can be controlled independently of corrugation. We have demonstrated that lipid bilayer membranes on solid surfaces experience forces that induce asymmetry in density and pressure profiles, with higher densities and pressures in the proximal leaflet of the supported bilayer. The concomitant membrane ordering constitutes a significant entropic penalty to bilayer binding, which substantially reduces the free energy of adsorption. It furthermore reduces interdigitation of the leaflets and-depending on how this is measuredaffects the bilayer thickness. It finally also abates large-scale membrane undulations, which is the dominant cause for an increased lateral expansion modulus. Owing to the very generic nature of our model, these observations are applicable to a wide range of membrane systems whose assemblies are driven by the amphiphilic nature of their constituents- such as lipids or block copolymers_-and which adsorb on a solid interface. The level of coarse graining and our use of a water-free model permits rapid computations and the ability to scale future simulations to sizes comparable to those accessible at the laboratory bench.

From an experimental point of view, we believe the most relevant lesson from our studies to be the following: in the light of the observed pronounced bilayer asymmetries it is highly desirable to conduct experiments which can probe the physical properties of the two inequivalent monolayers sepa- 
rately. If this is not possible, the bilayer-averaged data may still be best modeled as the superposition of two distributions, one for the upper and one for the lower leaflet. For the fluorescent techniques mentioned at the beginning of our paper, leaflet selectivity for diffusion and phase behavior measurements may benefit from controlled ion quenching of fluorescence in the distal leaflet, and from preferential partitioning of probes into the proximal and distal leaflet. When analyzing single particle tracking data one may try to model the data as overlap of two populations in terms of the diffusion constant or trapping likelihood. When analyzing the results for chain order measurements in NMR experiments, attempting to model the data as an average of two leaflets behaving differently may be beneficial and electron densities in $\mathrm{x}$ ray and neutron reflectivity experiments may show reduced interdigitation. Our observations point toward the effects that ought to be expected during such observations, and how they can be understood as a fairly natural reorganization of the proximal bilayer leaflet upon adsorption.

A natural extension of our investigations is the study of supported lipid bilayers at corrugation levels significantly larger than the coarse-grain particle size. In this case it will become important to tune the solid surface properties to match the macroscopic surface characteristics of specific classes of materials with respect to lipid solid interactions. These surface features will facilitate studies of membrane nanomechanics and the efficient energy calculations will provide an alternative to the mathematical challenges of analytical shape equations that similar treatments of membranes via continuum mechanics face.

\section{ACKNOWLEDGMENTS}

This project was supported by the NSF NIRT Program and a NSF-IREE international travel supplement (Grant No. CBET 0506662). M.I.H. also acknowledges support by an industry/campus supported fellowship under the Training Program in Biomolecular Technology (No. T32-GM08799) at the University of California, Davis.

${ }^{1}$ L. K. Tamm and H. M. McConnell, Biophys. J. 47, 105 (1985).

${ }^{2}$ E. Sackmann, Science 271, 43 (1996).

${ }^{3}$ T. V. Ratto and M. L. Longo, Biophys. J. 83, 3380 (2002).

${ }^{4}$ T. V. Ratto and M. L. Longo, Langmuir 19, 1788 (2003).

${ }^{5}$ W.-C. Lin, C. D. Blanchette, and M. L. Longo, Biophys. J. 92, 2831 (2007)

${ }^{6}$ M. C. Howland, A. W. Szmodis, B. Sanii, and A. N. Parikh, Biophys. J. 92, 1306 (2007).

${ }^{7}$ C. D. Blanchette, W.-C. Lin, T. V. Ratto, and M. L. Longo, Biophys. J. 90, 4466 (2006).

${ }^{8}$ J. V. Ricker, N. M. Tsvetkova, W. F. Wolkers, C. Leidy, F. Tablin, M. Longo, and J. H. Crowe, Biophys. J. 84, 3045 (2003).

${ }^{9}$ A. A. Brian and H. M. McConnell, Proc. Natl. Acad. Sci. U.S.A. 81, 6159 (1984)

${ }^{10}$ K. Mossman and J. Groves, Chem. Soc. Rev. 36, 46 (2007).

${ }^{11}$ C. D. Blanchette, W. C. Lin, C. A. Orme, T. V. Ratto, and M. L. Longo, Langmuir 23, 5875 (2007).

${ }^{12}$ C. E. Miller, J. Majewski, E. B. Watkins, D. J. Mulder, T. Gog, and T. L. Kuhl, Phys. Rev. Lett. 100, 058103 (2008).
${ }^{13}$ C. E. Miller, J. Majewski, T. Gog, and T. L. Kuhl, Phys. Rev. Lett. 94, 238104 (2005).

${ }^{14}$ G. Valincius, D. J. McGillivray, W. Febo-Ayala, D. J. Vanderah, John J. Kasianowicz, and M. Lösche, J. Phys. Chem. B 110, 10213 (2006).

${ }^{15}$ D. J. McGillivray, G. Valincius, D. J. Vanderah, W. Febo-Ayala, J. T. Woodward, F. Heinrich, J. J. Kasianowicz, and M. Lösche, BioInterphases 2, 21 (2007).

${ }^{16}$ T. M. Bayerl and M. Bloom, Biophys. J. 58, 357 (1990).

${ }^{17}$ H. Schonherr, J. M. Johnson, P. Lenz, C. W. Frank, and S. G. Boxer, Langmuir 20, 11600 (2004).

${ }^{18}$ W. Knoll, C. W. Frank, C. Heibel, R. Naumann, A. Offenhäusser, J. Rhühe, E. K. Schmidt, W. W. Shen, and A. Sinner, Rev. Mol. Biotechnol. 74, 137 (2000).

${ }^{19}$ J. C. Munro and C. W. Frank, Langmuir 20, 3339 (2004).

${ }^{20}$ J. C. Munro and C. W. Frank, Langmuir 20, 10567 (2004).

${ }^{21}$ K. Sengupta, J. Schilling, S. Marx, M. Fischer, A. Bacher, and E. Sackmann, Langmuir 19, 1775 (2003).

${ }^{22}$ G. W. Feigenson and J. T. Buboltz, Biophys. J. 80, 2775 (2001).

${ }^{23}$ W.-C. Lin, C. D. Blanchette, T. V. Ratto, and M. L. Longo, Biophys. J. 90, 228 (2006).

${ }^{24}$ R. Parthasarathy and J. T. Groves, Cell Biochem. Biophys. 41, 391 (2004).

${ }^{25}$ C. M. Ajo-Franklin, P. V. Ganesan, and S. G. Boxer, Biophys. J. 89, 2759 (2005).

${ }^{26}$ J. Korlach, P. Schwille, W. W. Webb, and G. W. Feigenson, Proc. Natl. Acad. Sci. U.S.A. 96, 8461 (1999).

${ }^{27}$ E. Kalb, S. Frey, and L. K. Tamm, Biochim. Biophys. Acta 1103, 307 (1992).

${ }^{28}$ M. Kuhner, R. Tampe, and E. Sackmann, Biophys. J. 67, 217 (1994).

${ }^{29}$ O. Prucker, C. A. Naumann, J. Rühe, W. Knoll, and C. W. Frank, J. Am. Chem. Soc. 121, 8766 (1999).

${ }^{30}$ W. W. Shen, S. G. Boxer, W. Knoll, and C. W. Frank, Biomacromolecules 2, 70 (2001).

${ }^{31}$ D. R. Heine, A. R. Rammohan, and J. Balakrishnan, Mol. Simul. 33, 391 (2007).

${ }^{32}$ S. V. Bennun, A. N. Dickey, C. Xing, and R. Faller, Fluid Phase Equilib. 261, 18 (2007).

${ }^{33}$ C. Xing and R. Faller, J. Phys. Chem. B 112, 7086 (2008).

${ }^{34}$ B. Rózycki, R. W. Thomas, and L. Reinhard, Phys. Rev. Lett. 100, 098103 (2008).

${ }^{35}$ I. R. Cooke, K. Kremer, and M. Deserno, Phys. Rev. E 72, 011506 (2005).

${ }^{36}$ G. Brannigan, P. F. Philips, and F. L. H. Brown, Phys. Rev. E 72, 011915 (2005).

${ }^{37}$ O. Farago, J. Chem. Phys. 119, 596 (2003).

${ }^{38}$ H. Noguchi and M. Takasu, J. Chem. Phys. 115, 9547 (2001).

${ }^{39}$ J. M. Drouffe, A. C. Maggs, and S. Leibler, Science 254, 1353 (1991).

${ }^{40}$ H.-J. Limbach, A. Arnold, B. A. Mann, and C. Holm, Comput. Phys. Commun. 174, 704 (2006)

${ }^{41}$ I. R. Cooke and M. Deserno, J. Chem. Phys. 123, 224710 (2005).

${ }^{42}$ J. D. Weeks, D. Chandler, and H. C. Andersen, J. Chem. Phys. 54, 5237 (1971).

${ }^{43}$ B. Sanii and A. N. Parikh, Soft Matter 3, 974 (2007).

${ }^{44}$ A. N. Dickey and R. Faller, Biophys. J. 92, 2366 (2007).

${ }^{45}$ M. Patra, I. Vattulainen, E. Salonen, E. Terama, R. Faller, B. W. Lee, J. Holopainen, and M. Karttunen, Biophys. J. 90, 1121 (2006).

${ }^{46}$ H. V. Ly and M. L. Longo, Biophys. J. 87, 1013 (2004).

${ }^{47}$ J. H. Irving and J. G. Kirkwood, J. Chem. Phys. 18, 817 (1950).

${ }^{48}$ O. H. S. Ollila, T. Rog, M. Karttunen, and I. Vattulainen, J. Struct. Biol. 159, 311 (2007).

${ }^{49}$ S. Ollila, M. T. Hyvonen, and I. Vattulainen, J. Phys. Chem. B 111, 3139 (2007).

${ }^{50}$ C. Xing, O. H. S. Ollila, I. Vattulainen, and R. Faller (unpublished).

${ }^{51} \mathrm{~J}$. Rowlinson and B. Widom, Molecular Theory of Capillarity (Dover, New York, 2002).

${ }^{52}$ E. Lindahl and O. Edholm, J. Chem. Phys. 113, 3882 (2000).

${ }^{53}$ F. David and S. Leibler, J. Phys. II 1, 959 (1991). 